\title{
Modelling and characterisation of geometric errors on 5-axis machine-tool
}

\author{
Fabien Viprey ${ }^{1,2, *}$, Hichem Nouira ${ }^{2}$, Sylvain Lavernhe ${ }^{1}$, and Christophe Tournier ${ }^{1}$ \\ ${ }^{1}$ LURPA, ENS Paris-Saclay, Univ. Paris-Sud, Université Paris-Saclay, 94235 Cachan, France \\ ${ }^{2}$ Laboratoire Commun de Métrologie (LNE-Cnam), 1, rue Gaston Boissier, 75724 Paris Cedex 15, France
}

Received: 7 January 2019 / Accepted: 16 May 2019

\begin{abstract}
This research work deals with the geometric modelling of 5-axis machine tool based on a standardised parameterisation of geometric errors with the aim to decrease the volumetric error in the workspace. The identification of the model's parameters is based on the development of a new standard thermo-invariant material namely the Multi-Feature Bar. Thanks to its calibration and a European intercomparison, it now provides a direct metrological traceability to the SI metre for dimensional measurement on machine tool in a hostile environment. The identification of three intrinsic parameters of this standard, coupled with a measurement procedure ensures a complete and traceable identification of motion errors of linear axes. An identification procedure of location and orientation errors of axes is proposed by probing a datum sphere in the workspace and minimising the time drift of the structural loop and the effects of the previously identified motion errors. Finally, the developed model partially identified, allows the characterisation of $95 \%$ of the measured volumetric error. Therefore, the mean volumetric error not characterised by the model only amounts to $8 \mu \mathrm{m}$.
\end{abstract}

Keywords: 5-axis machine tool / modelling / identification / geometric errors / material standard

\section{Introduction}

The multifunctional machine tools (MTs) named 5-axis MT are means of production frequently used in the manufacturing industry. However, in spite of an industrial need clearly expressed, the product range of available MTs on the market does not yet have the capability to perform traceable on-line measurement produced geometric entities. Although measurement is considered to be carried out on MT for the adjustment of production, or for the control of specifications with requirements compatible with the performance of MT, is not traceable. The measure obtained by a machine tool is often affected by the limitations of the integrated geometrical corrections in the Computer Numerical Control (CNC) and often obtained in different conditions from real conditions in a shop floor. Environmental factors, thermal errors, loads, dynamic errors in MT, countouring errors are parameters which limit the performance of MTs used as measuring system. With these parameters, it is currently not possible to rule on the accuracy and the uncertainty of measurement results. In this general context, the final goal of this paper aims to control the real geometry of the MT structure in order to improve, in the short term, the manufacturing process of workpieces. Finally, the main objective is to get MT with a level of accuracy lower

*e-mail: fabien.viprey@ensam.eu than $10 \mu \mathrm{m}$ in its workspace. This control of the geometry is based on a modelling and an identification of the real geometry of the structure in a quasi-static behaviour, which is similar to the structure behaviour associated with the measurement operations.

\section{Geometric errors}

A machine tool is a an assembly of kinematic groups and measuring systems which are an integral part of the structural loop. This structural loop is specified in [1] as the assembly of components that maintains the relative position between two specified objects. A typical pair of specified objects can be a cutting tool and a workpiece, for example.

The geometric errors are specified in $[1,2]$. They can be split up into two categories: motion errors of the axis $\left(E_{i j}\right.$, Fig. 1a (2) and the location and orientation errors of the axis $\left(E_{i 0 j}\right.$, Fig. 1a (3)).

5 -axis MT counts 30 motion errors (6 errors per axis) and 19 location and orientation errors of axes (4 per linear axis and 5 per rotary axis). The process introduces 12 geometric errors of assembly $d_{i w}$ or $t: 6$ components of tool positioning $(t)$ in the spindle and 6 components of the workpiece positioning $(w)$ on the table of the MT. In a quasi-static context, the geometrical errors are responsible of the volumetric error $\mathbf{V}_{\mathbf{C A X Y Z}}[3,4]$ defined as 


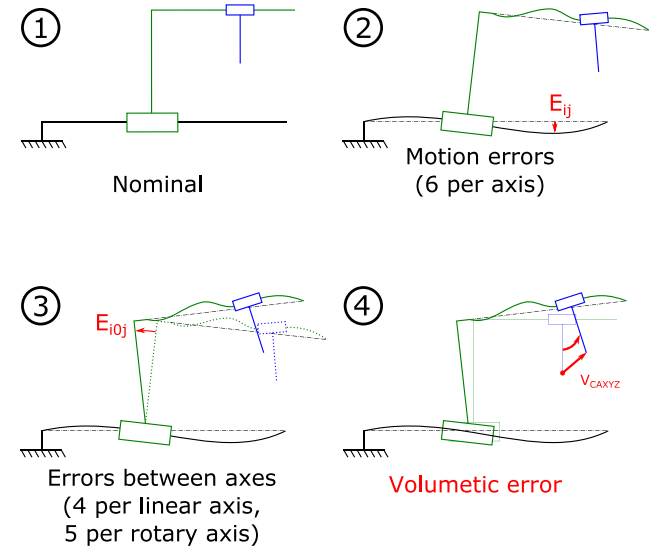

(a)

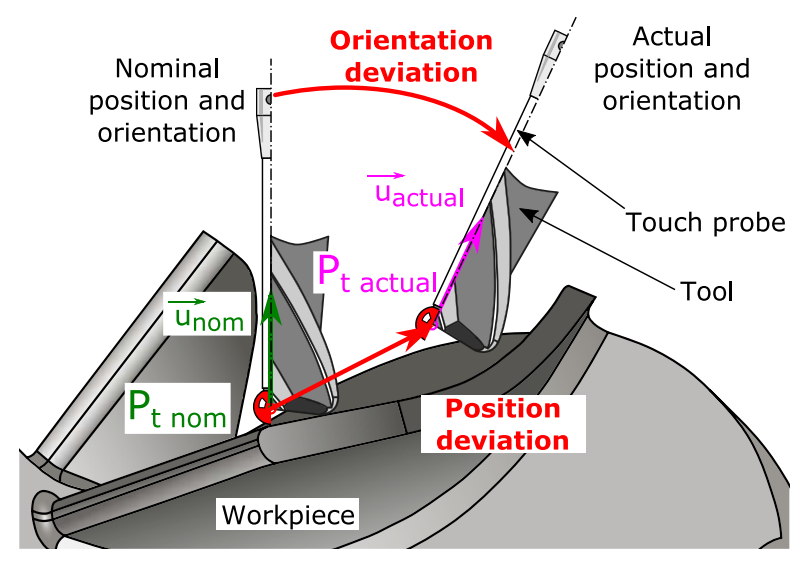

(b)

Fig. 1. Volumetric error $\mathbf{V}_{\mathbf{C A X Y Z}}$ in MT: consequence of the geometric errors $E_{i j}$ and $E_{i 0 j}$. (a) Illustrations of the motion errors and the location and orientation errors of axis. (b) Volumetric error in the context of manufacturing or measurement on 5-axis MT.

the relative deviation between the tool and the workpiece expressed in the workspace (Fig. 1b) in more than $70 \%$.

\section{Geometric model}

In order to identify the geometric errors - the main sources of the measured volumetric error $\mathbf{V}_{\mathbf{C A X Y z}}$ between the effective geometry and the nominal geometry - a modelling of the biased and nominal behaviour of the structural loop is proposed. In the case of a quasi-static and infinitely rigid body behaviour of the structural loop, this modelling rests on the specified definition of the geometric errors and on homogeneous transformation matrix formalism. The modelling is then applied to a 5-axis MT Mikron UCP710 with the [w C' A' b X Y Z (C1) t] structural loop, according to [5]. By difference of the TCP coordinates in the workspace expressed by using both models (i.e. biased model is the minuend and nominal model is the subtrahend), it is possible to calculate the volumetric error $\mathbf{V}_{\mathbf{C A X Y Z}}$ in the workspace according to the intrinsic geometric parameters of the structure $\boldsymbol{\xi}$, the geometric parameters of the tool $\boldsymbol{\tau}$, the geometric parameters relative to the workpiece setting-up $\boldsymbol{\psi}$, q-vector of the articular parameters, as well as the $\mathbf{E}$-vector of geometric errors (Eq. (1)).

$$
\begin{aligned}
\mathbf{V}_{\mathbf{C A X Y Z}} & =\left(\begin{array}{l}
\overrightarrow{P_{\text {tnom }} P_{\text {tactual }}} \\
\mathbf{u}_{\text {nom }}-\mathbf{u}_{\text {actual }}
\end{array}\right)_{6 \times 1} \\
& =[\delta x \delta y \delta z \delta i \delta j \delta k]^{T}=\mathbf{f}(\boldsymbol{\xi}, \boldsymbol{\tau}, \boldsymbol{\psi}, \mathbf{q}, \mathbf{E})
\end{aligned}
$$

A volumetric error in the workspace from a targeted nominal position can be deduced from the measurement of the position in the workspace of material standards or artefacts. This procedure is classically used on MT [6] because it is easy to perform and leads to a short acquisition chain limited to the workspace, which minimises the influence of other sources of errors. Furthermore, the result of measurement is directly equal to the components of the volumetric error. Therefore, from an operational point of view, the result is directly linked to the effect of geometric errors on the geometry of the produces or measured workpieces.

However, the measurement is totally disconnected from articular parameters, which are, like the geometric errors, inherent to the structural loop. To have an image as faithful as possible of this loop, the strategy is to model the geometric bias as an articular deviation generated by the geometric errors. This modelling is based on the fact that the position of measured point is constantly nominal in the workspace, in other words, the volumetric error is equal to zero (for every measured point, $\mathbf{V}_{\mathbf{C A X Y Z}}=\mathbf{0}$ ). Then, the geometrical bias $\boldsymbol{\delta} \mathbf{q}_{\mathbf{m o d}}$ is defined as the difference between the deviated articular configuration, and the nominal articular configuration calculated from the model (Fig. 2). After first order linearisation of the geometric bias with hypotheses of small displacements (i.e. small rotations), this difference is written as the product of a matrix of sensitivity $\mathbb{S}$ by the E-vector of errors (Eq. (2)). The linearisation was validated by the development of a virtual MT (VMT) designed as a tool to display and understand the volumetric error effect in the workspace. As a matter of fact, the effect of linearisation is qualified and quantified by comparison of the volumetric error calculated from the complete model and the volumetric error calculated from the linearised model. The values of E-vector of errors have been chosen with regard to the geometric errors identification in the scientific literature.

$$
\begin{aligned}
\boldsymbol{\delta} \mathbf{q}_{\text {mod }} & =\left(\begin{array}{l}
\delta X_{\text {mod }} \\
\delta Y_{\text {mod }} \\
\delta Z_{\text {mod }}
\end{array}\right)=\left(\begin{array}{c}
X_{\text {biased }}-X \\
Y_{\text {biased }}-X \\
Z_{\text {biased }}-Z
\end{array}\right)=\mathbb{S} \times \mathbf{E}, \text { or } \\
\mathbb{S} & =\mathbf{g}(\boldsymbol{\xi}, \boldsymbol{\tau}, \boldsymbol{\psi}, \mathbf{q}=(A, C))
\end{aligned}
$$

\section{Design and production of equipment}

From the measurement of the geometric bias (Eq. (2)), it is possible to identify the vector $\mathbf{E}$, and therefore to reduce the geometric bias and a fortiori the volumetric error of the structural loop. This measurement is based on the acquisition of the articular positions of axes for a point with a long-lasting position in the workspace. 


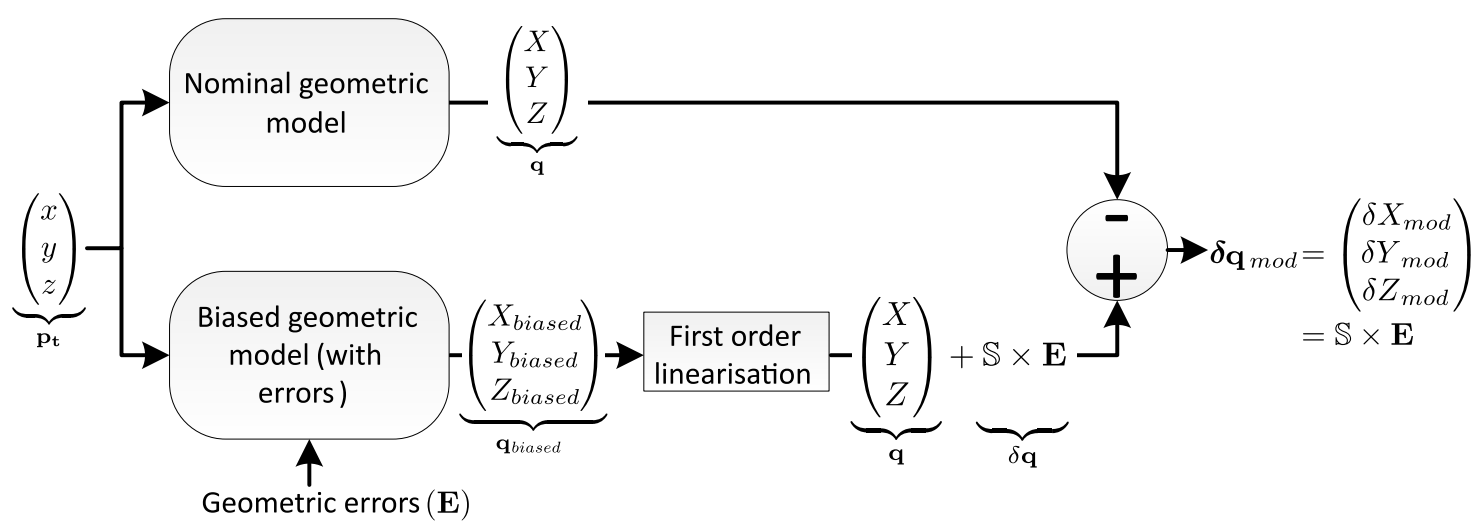

Fig. 2. Definition of the modelled geometric bias $\boldsymbol{\delta} \mathbf{q}_{\text {mod }}$.

\subsection{A novel real time measuring system of articular parameters}

The deviation of articular configuration is directly measured on the linear and angular measuring system during the trigger of accurate 3D touch probe used as a detector of zero. The nominal articular configuration is deducted from the nominal geometric model of the structural loop.

The deviation of articular configuration is obtained by using the on-board measuring systems in the MT: a touch probe and the measuring system (rules). Hardware architecture by the physical integration of a high accurate $3 \mathrm{D}$ touch probe $(2 \sigma=0.25 \mu \mathrm{m}$ at $240 \mathrm{~mm} / \mathrm{min})$ within the MT allows the measurement of points in the workspace. Following this hardware integration, software is developed to synchronise in real time (resolution $=10 \mathrm{~nm}$, frequency of acquisition $=33 \mathrm{kHz}$ ) the state of the stylus and the information from encoders of the linear and angular measuring system. After performing the procedure to acquire the machine zero point by counting distance-coded reference marks, the objective is to obtain the raw and absolute articular position from axes without any compensation or processing performed by the industrial CNC. The architecture of the measuring system is called LURPAlpeur (Fig. 3). LURPAlpeur is intended as an autonomous measuring system, irrespective of the behaviour of the existing industrial CNC. After the qualification of this new measuring device, an articular position measurement can be obtained. The estimated uncertainty of this measurement due to a delay in synchronism is equal to $0.2 \mu \mathrm{m}$ at $100 \mathrm{~mm} / \mathrm{min}$ and the repeatability of measure amounts to $0.97 \mu \mathrm{m}$.

\subsection{A novel material standard: the Multi-Feature Bar (MFB)}

For a given position in the workspace, the validation of the volumetric error condition equal to zero is made possible by using a material standard connected to the SI metre definition. According to a review of the existing material standards used on MT or on CMM and their advantages and disadvantages, a new material standard was proposed: the Multi-Feature Bar (MFB). Its physical realisation in Invar material (ratio $\alpha / \lambda=7.7 \times 10^{-8} \mathrm{~m} \mathrm{~W}^{-1}$ ) guarantees a thermo-invariance of its geometry. The MFB is a succession of elementary patterns including geometrical features such as plans and cylinders (Fig. 4) ensuring the identification of 3 local intrinsic parameters in the local frame of the MFB: a linear positioning error $E_{x x_{\mathrm{MFB}}}$ and two straightness errors $E_{y x_{\mathrm{MFB}}}$ and $E_{z x_{\mathrm{MFB}}}$, for a single positioning of the MFB in the workspace of MT [7]. The[24.6pc] isostatic setting-up by minimising the deflections of the MFB, as well as the clamping system provide flexibility to the operator regarding the MFB positioning in the workspace. The geometry of the MFB ensures a total and bilateral accessibility of the geometric features of patterns. Thanks to this advantage, a calibration is performed by a reversal technique on an accurate CMM connected to the SI metre definition. This method is carried out to separate the geometric errors from the CMM and the geometric errors from the MFB with the aim to delete the residual errors of measurement during the calibration. These residual errors of measurement are essentially due to the residual geometric errors of the CMM. Compensation of the geometric errors in the case of the linear positioning error calibration is carried out, because the separation of the linear positioning error of the CMM from those of the MFB is not possible by using the reversal technique.

In addition, in the context of the European project IND62: JRP-TIM, an intercomparison was organised to assure a sufficient level of confidence of the MFB calibration at LNE, and to estimate the reproducibility of the calibration method. The participation of various national metrology institute in this European intercomparison demonstrate the efficiency of the MFB, designed and produced at LNE as well as the control of its calibration by the data processing of measurement results. At this stage of the study, subject to conservation of a material standard [8], LNE has a thermo-invariant material standard which provides three intrinsic parameters contrary to available hole bars on the market. In conclusion, by its metrological concepts, its geometry, and its calibration, the MFB is now recognised at European level.

\section{Identification of model parameters}

The direct measurement of the linear and angular measuring system by using the LURPALpeur for points with 


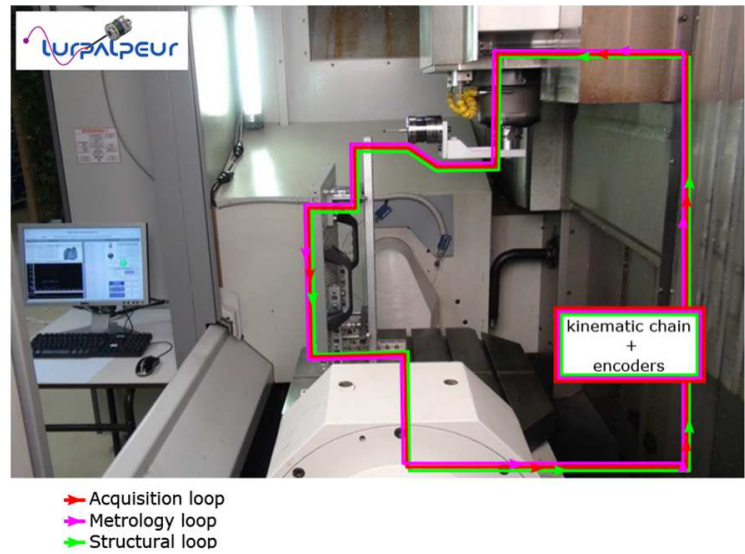

(a)

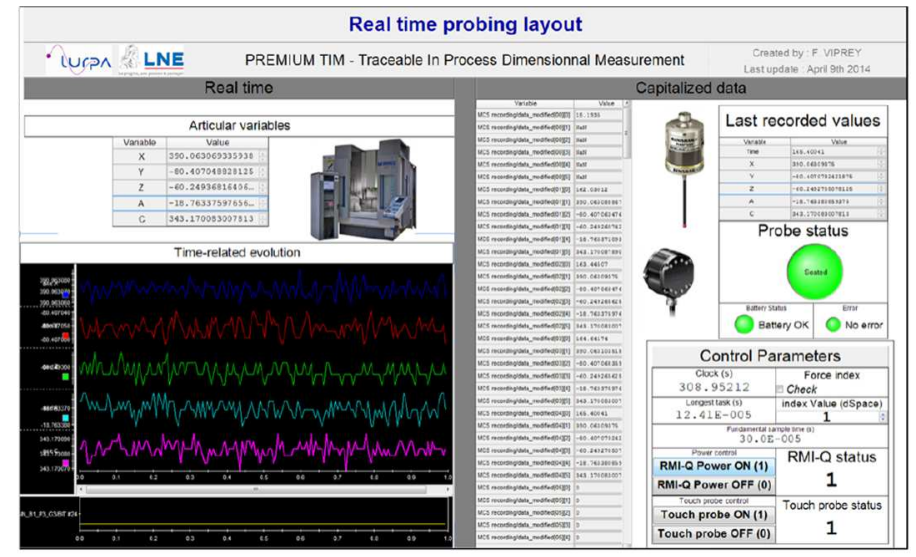

(b)

Fig. 3. Experimental device on MT: the LURPAlpeur (a) measuring device. (b) Real time human-computer interface.

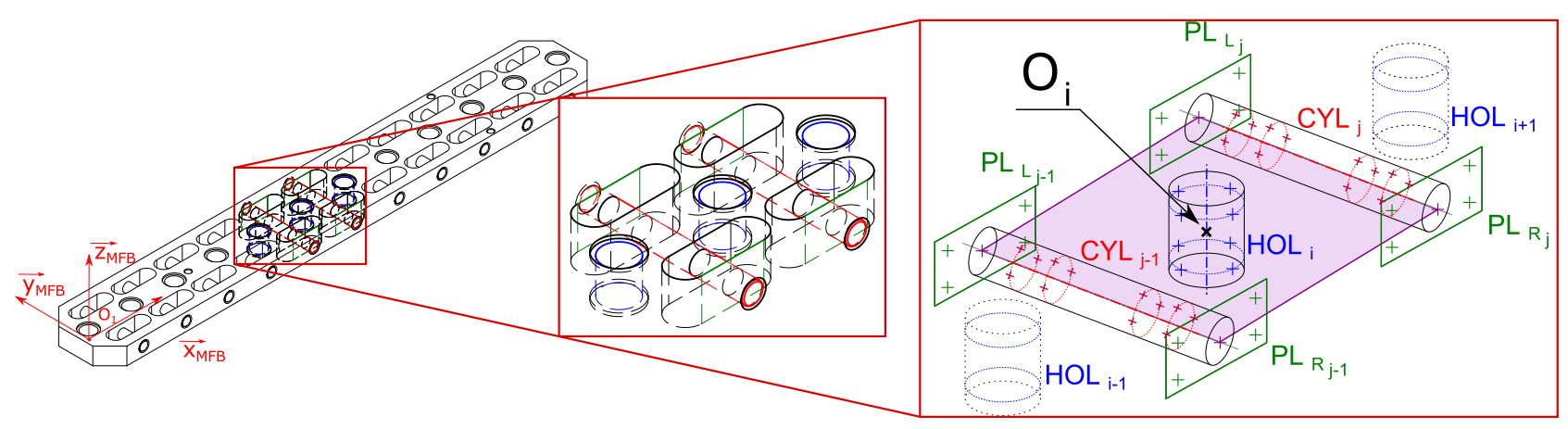

Fig. 4. Multi-Feature Bar: 3D CAD model, detail of the pattern and construction of the point of interest $O_{i}$.

positions supposed to be invariant in the workspace, gives access to the measured geometric bias (3).

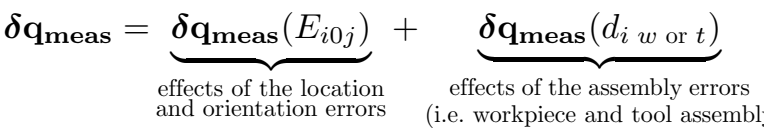

$$
\begin{aligned}
& \text { at the ends of structural loop) } \\
& +\underbrace{\boldsymbol{\delta} \mathbf{q}_{\text {meas }}\left(E_{i j}\right)}_{\begin{array}{c}
\text { effects of the } \\
\text { motion errors }
\end{array}}+\underbrace{\boldsymbol{\delta} \mathbf{q}_{\text {meas }}(\epsilon)}_{\begin{array}{c}
\text { effects of the } \\
\text { random errors }
\end{array}}
\end{aligned}
$$

This equation shows the dependence between the effects of the geometrical errors on the measured geometric bias $\boldsymbol{\delta} \mathbf{q}_{\text {meas. }}$. For the sake of clarity, the term of interdependence (i.e. interaction between errors) is not mentioned. However, this dependence was mathematically explained.

To minimise the interdependence between the geometrical errors, the identification procedure is divided into two main activities:

- the identification of motion errors by 11 measurements for 8 positionings of the MFB in the workspace (reduced to 5 by using rotary axes) (Fig. 5a). The identification is detailed in [7], and identified parameters are presented in Table 1. The motion errors' values are identified on each point of interest $O_{i}$ (i.e. each $50 \mathrm{~mm}$ ). The data are interpolated by $C^{2}$ interpolating cubic spline models, leading to provide accurate compensation models;

- after motion errors compensation and temporal drift compensation, the identification of errors of location and orientation of the axis as well as assembly errors is carried out by a datum sphere probing in 50 different articular configurations distributed in the workspace (Fig. 5b). The identified parameters are depicted in Table 1.

The identification process by using datum sphere probing has been previously validated on Virtual MT (VMT). This VMT is used to control the quality of the identification process. Moreover, the reduction of model to obtain the minimum number of model parameters to depict the effects of position and axis orientation errors is performed by using this VMT. An analysis of the sensitivity of parameters is carried out in order to adjust the measurement procedure (e.g. number of measured articular configurations, the position of the datum sphere on the rotary table). Moreover, the effects of noise and numerical errors on the identification are calculated. The quality of identification is evaluated by using the identification residue $\mathbf{r}(\widehat{\mathbf{E}})$ illustrated in Figure 6 , as the difference between the measured geometric bias $\boldsymbol{\delta}_{\mathbf{q m e s}}$ 


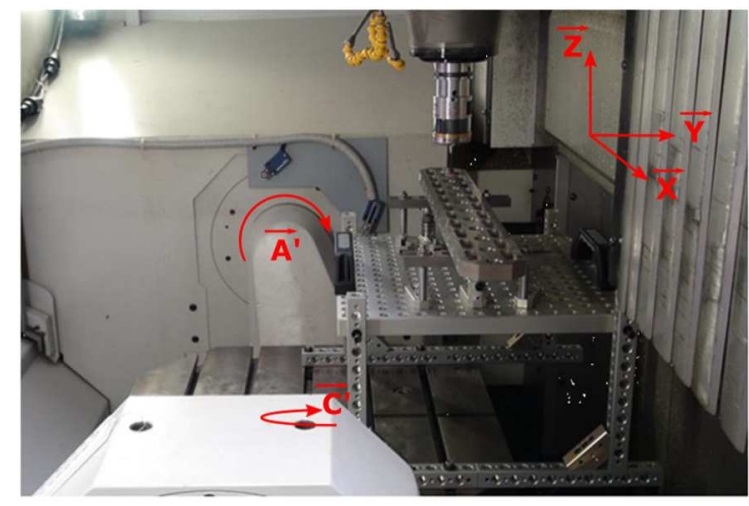

(a)

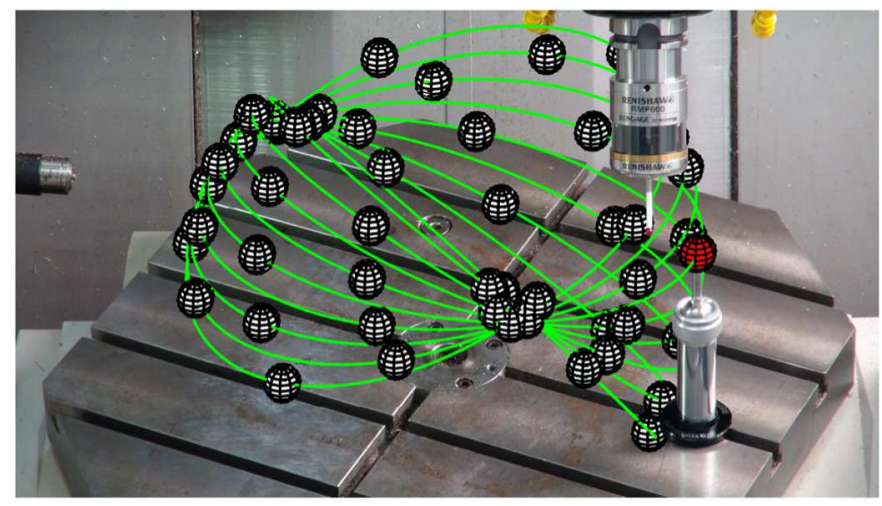

(b)

Fig. 5. Identification of geometric errors in the structural loop of the Mikron UCP710. (a) Identification of motion errors on three linear axes thanks to the MFB. (b) Identification of location and orientation errors as well as assembly errors thanks to datum sphere probing.

Table 1. Geometric errors identified on the 5-axis machine-tool.

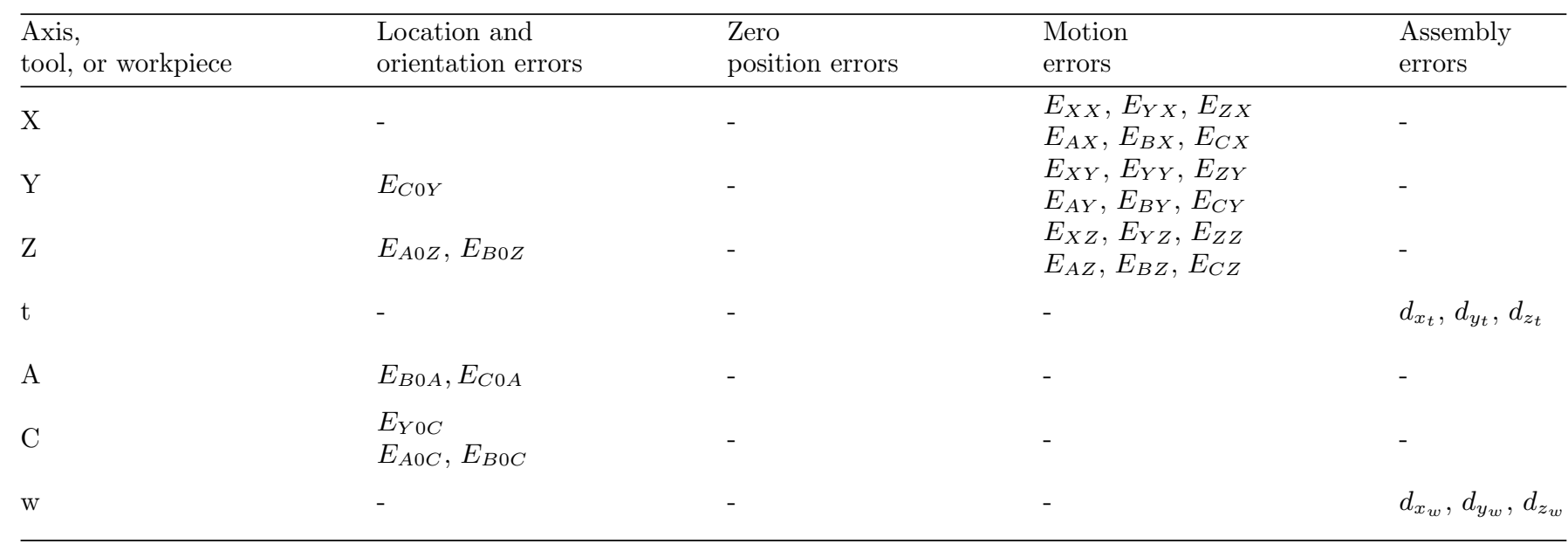

and the modelled bias $\boldsymbol{\delta} \mathbf{q}_{\mathbf{m o d}}$ after first order simplification. It quantifies the part of the measured geometric bias that is characterised by the first order effects of the geometric errors identified $\widehat{\mathbf{E}}$. The value of the residue may be due to the linearisation effects of the first-order model (i.e. limit of the small rotations hypothesis), the dynamic effects of the structural loop, its thermo-mechanical load and the environmental shop floor conditions.

Nevertheless, the term $\boldsymbol{\delta} \mathbf{q}_{\text {meas }}(\epsilon)$ which expresses the effects of the zero mean random error (Eq. (3)) is minimised by repeating the measurements over several days to qualify the quasi-rigid behaviour [6] of the structural loop. The repeatability and reproducibility of measurement is characterised by the repetition of measurements which are necessary for the expression of uncertainty in measurement. The latter is also developed to quantify the effect of uncertainty in error identification and therefore the effect on identified volumetric error by using identified model. In the long term, this uncertainty of identification will be the base for the quantification of uncertainty in in situ or in-process measurement on MT connected with the SI metre definition.

\section{Results and discussion}

The motion errors identification procedure requires one day. On the one hand, the entire automated measurement of the MFB requires 13 min to probe 316 points for each position of MFB. On the other hand, the identification of location and orientation errors of axes and assembly errors required $52 \mathrm{~min}$ due to the probing of datum sphere for 50 articular configurations. The identification of location and orientation errors of axes and assembly errors is repeated 10 times over two days to take into account the reproducibility of the axes positioning. The entire measured geometric bias $\boldsymbol{\delta} \mathbf{q}_{\text {meas }}$ is deduced from the fitted centre of all associated spheres. Variations of components and the norm of the geometric bias are depicted in Figure 7a. The variations are due to the position of datum sphere in workspace for each articular configurations. Indeed, the visible oscillations are due to the effects of location and orientation errors of rotary axis (i.e. rotary table). The error bars reflect the dispersion due to the reproducibility of the measurement procedure. The mean value of the norm of measured geometric bias is equal to $118 \mu \mathrm{m}$ and its maximum value is equal to $191 \mu \mathrm{m}$ 


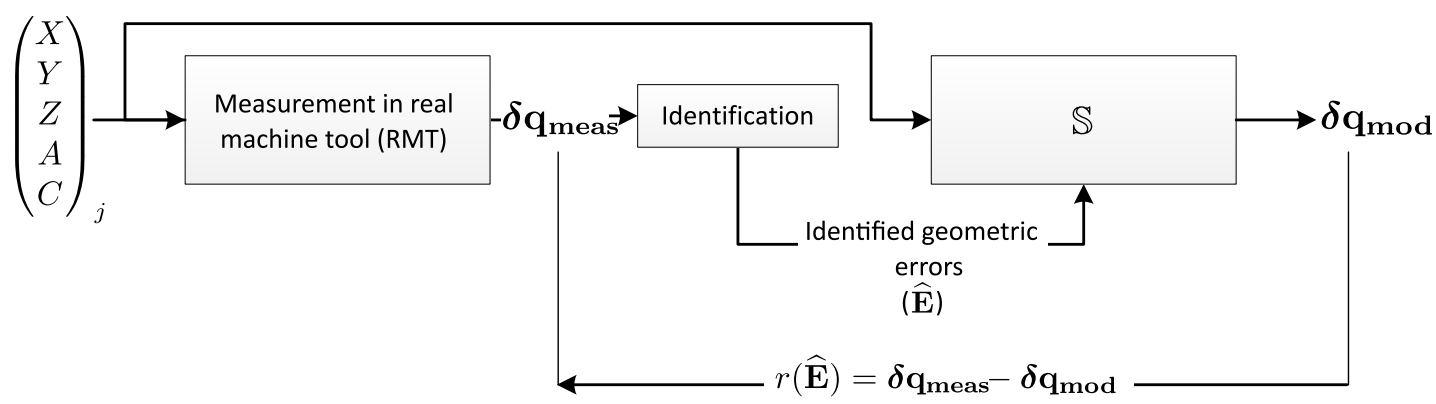

Fig. 6. Residue of identification $\mathbf{r}(\widehat{\mathbf{E}})$.

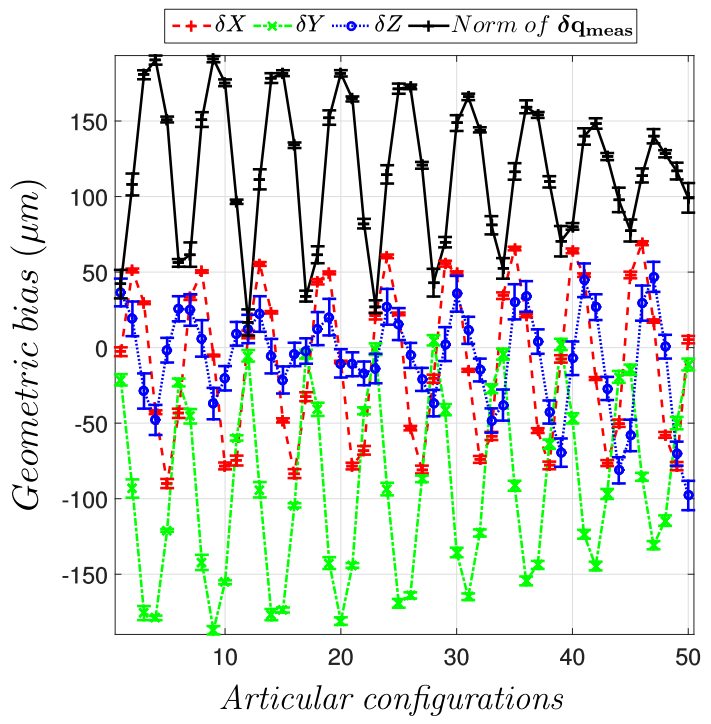

(a)

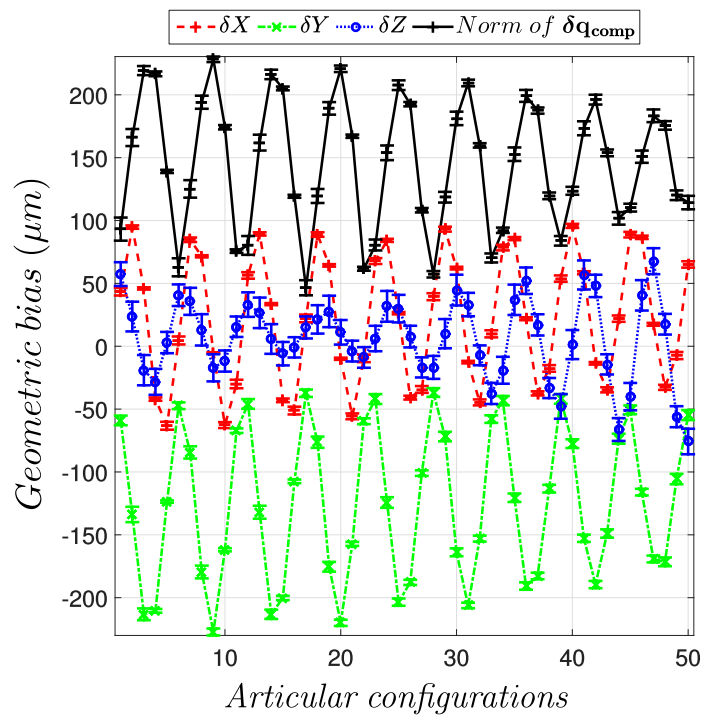

(b)

Fig. 7. Impact of the compensation of motion errors effects on $\boldsymbol{\delta} \mathbf{q}_{\text {comp }}$. (a) Components and norm of $\boldsymbol{\delta} \mathbf{q}_{\text {meas }}$ before motion errors compensation. (b) Components and norm of $\boldsymbol{\delta} \mathbf{q}_{\text {comp }}$ after motion errors compensation.

on the workspace. After this first identification location and orientation errors of axes, the modelled geometric bias induced by motion errors of linear axes (i.e. $\boldsymbol{\delta} \mathbf{q}_{\bmod }\left(E_{i j}\right)$ ) is compensated for the initially measured geometric bias $\boldsymbol{\delta} \mathbf{q}_{\text {meas }}$ (Eq. (4)). The resulting geometric bias, named $\delta \mathbf{q}_{\text {comp }}$ is depicted in Figure $7 \mathrm{~b}$. The mean normed value of compensated geometric bias $\boldsymbol{\delta} \mathbf{q}_{\text {comp }}$ without effects of motion errors of linear axes and temporal drifts is equal to $145 \mu \mathrm{m}$ and the maximum value is equal to $228 \mu \mathrm{m}$.

$$
\delta \mathbf{q}_{\text {comp }}=\delta \mathbf{q}_{\text {meas }}-\delta \mathbf{q}_{\text {meas }}\left(E_{i j}\right)
$$

The quality of identification evaluated by using the identification residue $\mathbf{r}(\widehat{\mathbf{E}})$ is shown in Figure 8a; the modelled geometric bias $\boldsymbol{\delta}$ qmod (Fig. 6) can be used to characterise $90 \%$ of the measurement $\left(\boldsymbol{\delta} \mathbf{q}_{\text {meas }}\right)$. Measurement are also performed with cold MT. It must be underlined that the MT shall be warmed up, because a test on cold MT (conditions of use not originally intended by the MT producer) generates an incorrect identification since the mean value of the norm of the residue is equal to $48 \mu \mathrm{m}$. In the case of warmed-up structural loop, the mean value of the norm of the residue is equal to $11.5 \mu \mathrm{m}$ (Fig. 8a). This residue correspond with the measured geometric bias by datum sphere probing before compensation of geometric bias induced by motion errors of linear axes.

The identification residue in Figure $8 \mathrm{~b}$ is calculated after compensation of motion errors. Thus, the residue only corresponds with the effect of location and orientation errors of axes, motion errors of rotary axes, thermal drifts and other sources of random errors assuming the formulation in equation (3). It is interesting to note a better characterisation of the geometric bias $\delta \mathbf{q}_{\text {comp }}$, since the identification residue decreases between the identification before and after compensation of motion errors effects. Indeed, a reduction of $3.4 \mu \mathrm{m}$ on the mean of the norm of the residue is observed between Figure $8 \mathrm{a}$ and b. The distribution of residue values is centered on $8 \mu \mathrm{m}$ and 45 configurations with a norm of residue inferior to $11 \mu \mathrm{m}$, whereas previously, the distribution of values was centered on $11 \mu \mathrm{m}$ and 45 configurations inferior to $18 \mu \mathrm{m}$.

Overall, once the effects of the motion errors are compensated, the identification procedure assures an average characterisation of the measured geometric bias equal to $94.5 \%$. Moreover, the differences between the components 

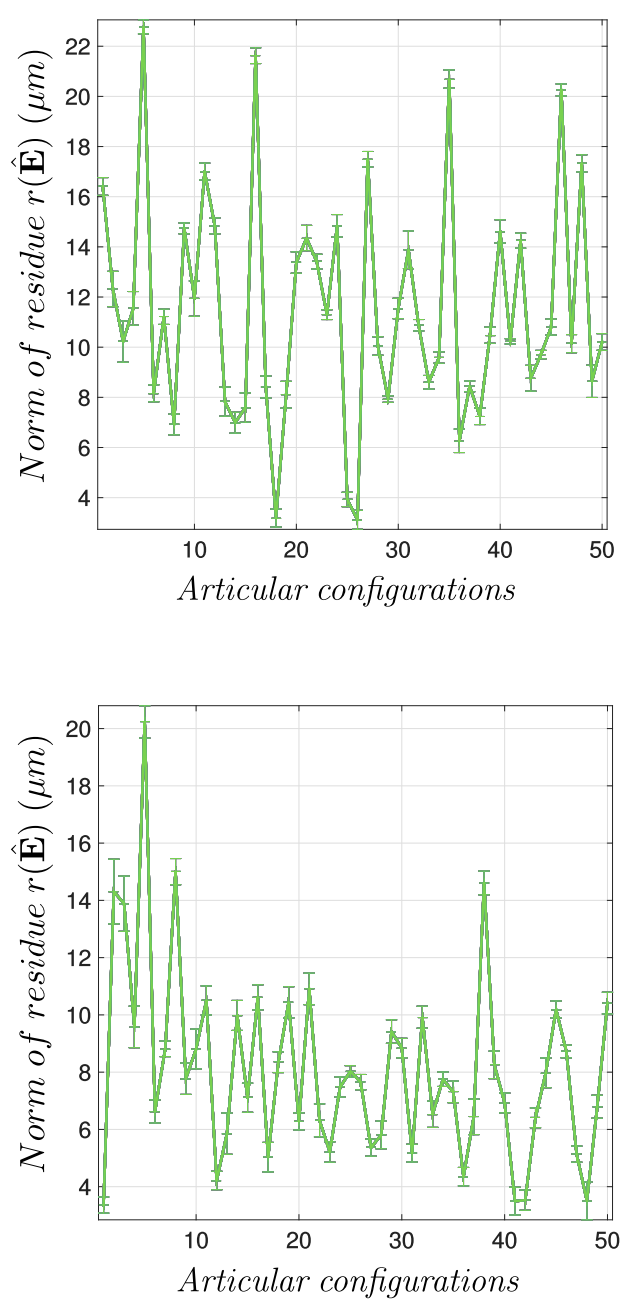

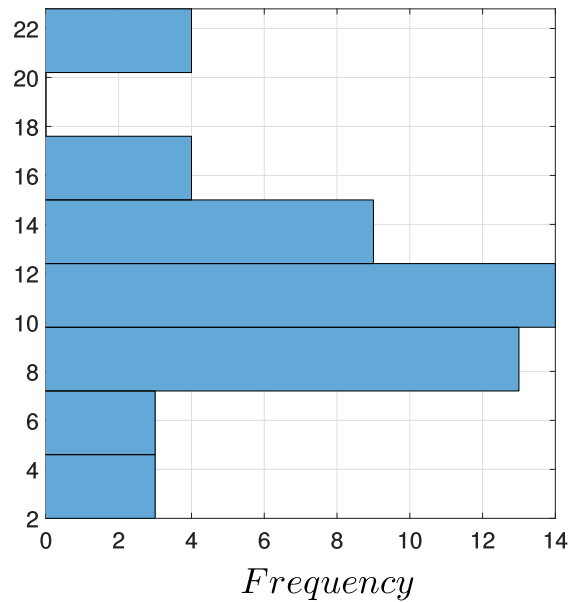

(a)

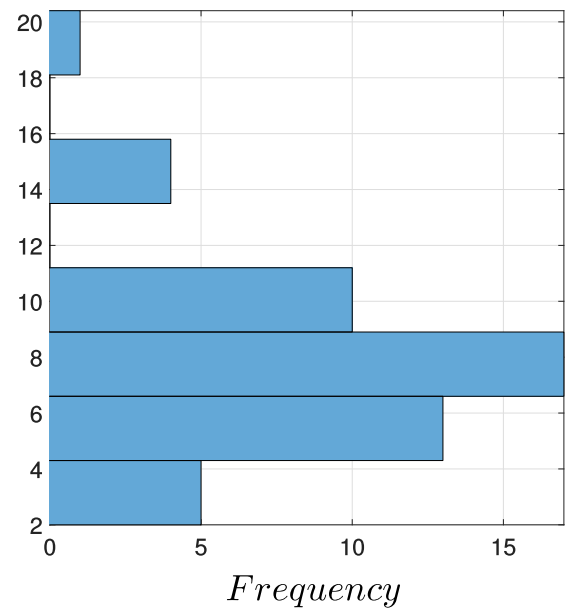

(b)

Fig. 8. Norm of the residue after identification. (a) Norm of residue $\|\mathbf{r}(\widehat{\mathbf{E}})\|$ after the identification and before motion errors compensation. (b) Norm of residue $\|\mathbf{r}(\widehat{\mathbf{E}})\|$ after the identification and after motion errors compensation.

of the measured geometric bias and the components of the modelled geometric bias do not exceed $3 \mu \mathrm{m}$, indicating a reliable characterisation of this measured bias by the model.

\section{Conclusion}

Research work on the modelling and characterisation of the real geometry of a structural loop is directly related to the industrial needs to have means of production with very high volumetric capability in both the productivity and the quality of the manufactured complex parts which are controlled by on-line traceable measurement. To achieve the objective of a volumetric error inferior to $10 \mu \mathrm{m}$, this work is oriented in order to solve the following scientific issue:

How to model and identify the actual geometry of a structural loop from measurement procedures easy to implement in a production flow, while assuring the metrological traceability of the measurement, in order to model the volumetric error as accurately as possible?
This work, by the developed model, and partially identified (identification of the motion errors of linear axes as well as location and orientation errors of axes), provide the characterisation of $95 \%$ of the measured geometric bias. Thus, the mean value of measured geometric bias not characterised by the identified model is equal to $8.1 \mu \mathrm{m}$, while the volumetric performances indicated by the MT manufacturer upon receipt of the 5-axis MT only amounted to $50 \mu \mathrm{m}$.

Acknowledgement. This work was funded through the European Metrology Research Programme (EMRP) Project IND62: JRP-TIM (Traceable In-process dimensional Measurement). The authors sincerely thank the EMRP organisation. The EMRP is jointly funded by the EMRP participating countries within EURAMET and the European Union (IND62: JRP-TIM).

\section{References}

[1] NF ISO 230-1:2012, Test code for machine tools - Part 1: Geometric accuracy of machines operating under no-load or quasi-static conditions 
[2] NF ISO 230-7:2007, Test code for machine tools - Part 7: Geometric accuracy of axes of rotation

[3] R. Ramesh, M. Mannan, A. Poo, Error compensation in machine tools - a review: Part I: geometric, cutting-force induced and fixture-dependent errors, Int. J. Mach. Tools Manuf. 40, 1235-1256 (2000)

[4] L. Andolfatto, S. Lavernhe, J. Mayer, Evaluation of servo, geometric and dynamic error sources on five-axis highspeed machine tool, Int. J. Mach. Tools Manuf. 51, 787-796 (2011)

[5] NF ISO 10791-6:2014. Test conditions for machining centres - Part 6: Accuracy of speeds and interpolations
[6] H. Schwenke, W. Knapp, H. Haitjema, A. Weckenmann, R. Schmitt, F. Delbressine, Geometric error measurement and compensation of machines - an update. CIRP Ann. Manuf. Technol. 57, 660-675 (2008)

[7] F. Viprey, H. Nouira, S. Lavernhe, C. Tournier, Novel multi-feature bar design for machine tools geometric errors identification, Prec. Eng. 46, 323-338 (2016)

[8] JCGM 200, International vocabulary of metrology - Basic and general concepts and associated terms (VIM) - 3rd edition BIPM, BIPM, 2012

Cite this article as: F. Viprey, H. Nouira, S. Lavernhe, and C. Tournier, Modelling and characterisation of geometric errors on 5-axis machine-tool, Mechanics \& Industry 20, 605 (2019) 